\title{
Advertising as information
}

Next time you hear a tobacco industry representative extolling the neo-classical economists' defense of tobacco advertising as "information", here's a good example to put the claim to the test. The ad for Imperial Tobacco's Embassy was published in the scatological British comic Viz, immensely popular particularly among 14-year-old schoolboys who like reading about the expletive-deleted adventures of characters such as Johnny Fartparts and Buster Gonad, a boy with enormous testicles. The written text of the ad: "Tar, but no tar very much" (translation: "thank you, but no thank you very much") simultaneously alludes to the brand's low-tar status and to the veterinarian's request to be given assistance in his search inside the cow's rear end.

But it is beside the point to deconstruct the ad for its propositional forms. Aside from the concern that this ad falsely suggests that the brand has "no tar", its semiotics offer little to the reader but an arresting picture. Rather, it would "work" on a teenage readership by being located in a magazine that their parents would disapprove of. There is a perfect apposition between the clandestine possession of the magazine, perhaps secreted under the mattress, and the equally disapproved cigarette pack. For the schoolboy intent on badging himself as an officinado of all that is reviled by fun-banishing parents, the magazine and Embassy are thus positioned as essential gear. The ad illustrates perfectly how totally beside the point limited rules in voluntary codes are when they confine themselves to proscriptions about not depicting any scenes that "glamorise" smoking or other naive descriptors. As media guru Marshall McLuhan reminded us: the medium is the message.

SIMON CHAPMAN

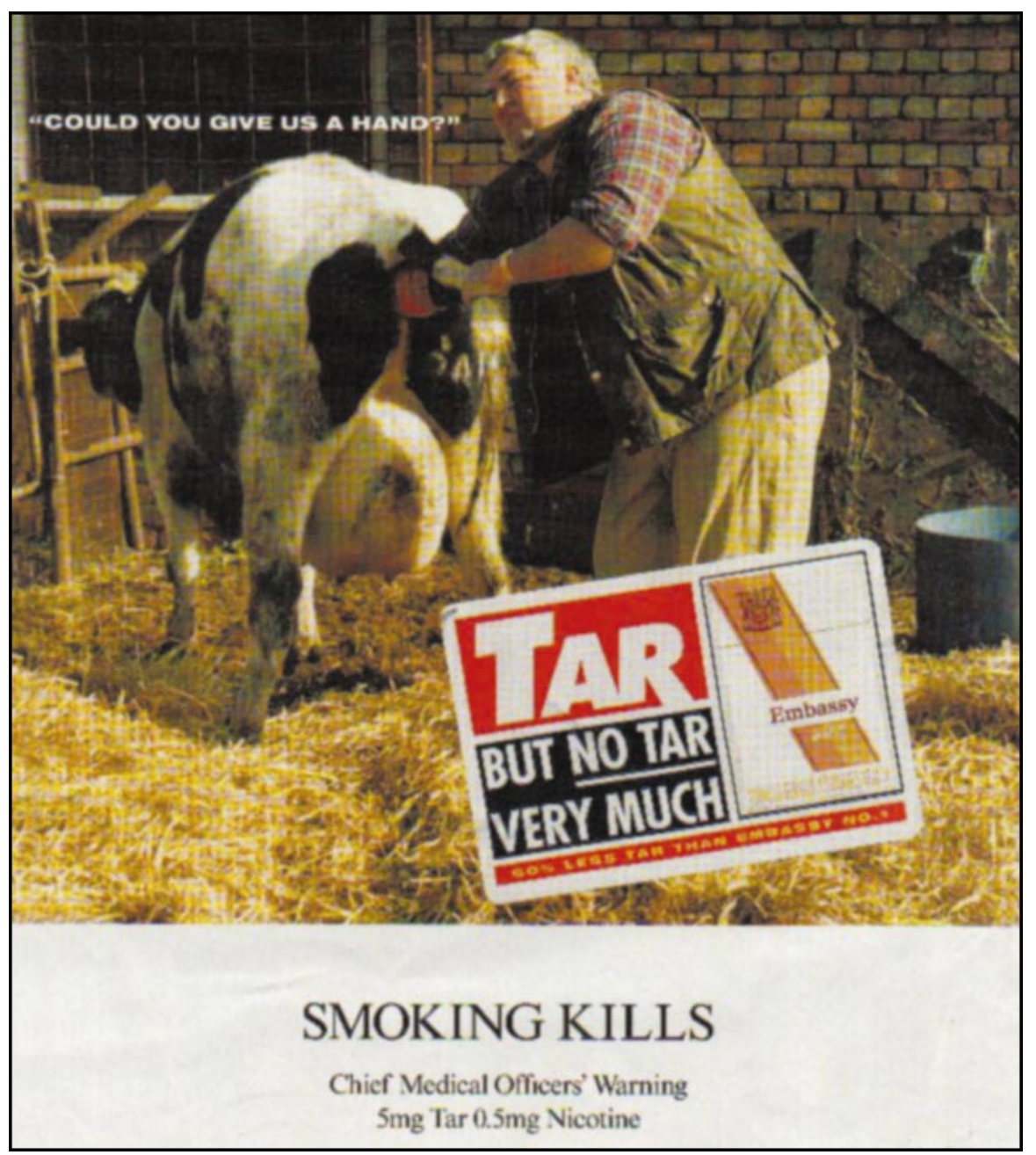

